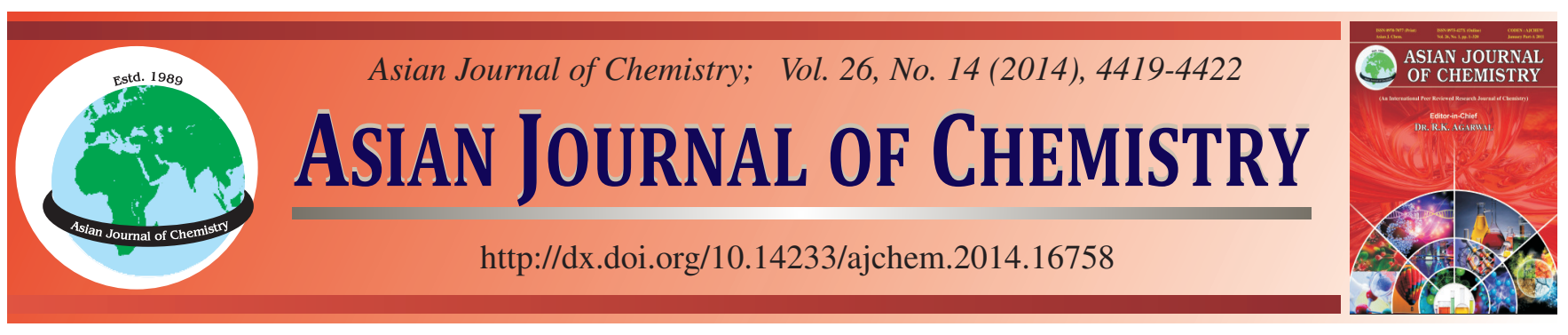

\title{
Preparation and Thermal Behavior of Novel Sulfur-Nitrogen Flame Retardant Containing Triazine Ring for Cotton Fabrics
}

\author{
Zhi-Yi ZhANG, Gui-Zhe ZhaO and YA-QING LiU*
}

Research Center for Engineering Technology of Polymeric Composites of Shanxi Province, College of Materials Science and Engineering, North University of China, Taiyuan 030051, P.R. China

*Corresponding author: E-mail: zhiyzhang@ sohu.com

Received: 26 November 2013;

Accepted: 25 January 2014;

Published online: 5 July 2014;

AJC-15487

A novel sulfur-nitrogen flame retardant containing triazine ring $(\mathrm{SN})$ has been synthesized and structurally characterized by elemental analysis, FT-IR and ${ }^{1} \mathrm{H}$ NMR spectroscopies. Meanwhile, the effects of reaction solvent, acid-binding agent, reaction temperature and molar ratio of starting materials on the yield of SN were investigated. Experiments showed that when the molar ratio of intermediate $\mathrm{M}$ to diethanol amine is 1 to 1 in the presence of sodium carbonate as acid-binding agent in water at $40{ }^{\circ} \mathrm{C}$, the yield of the obtained $\mathrm{SN}$ reaches $85 \%$. In addition, to evaluate the potential flame-retardant ability of the target flame retardant (SN), the thermal behaviors of SN, pure cotton, and cotton treated with SN were studied by thermogravimetry analysis. This result demonstrated that SN was a good charring agent and obviously improved the char-forming ability and the thermal stability of cotton fabrics.

Keywords: Sulfur-nitrogen, Flame retardant, Preparation, Cotton fabrics, Thermal behavior.

\section{INTRODUCTION}

In the past decades, the formaldehyde- or halogen-containing flame retardants have shown remarkable flame retardancy. But, they may lead to the environmental problems by generating large amounts of toxic and corrosive fumes during combustion and are restricted applications in many fields ${ }^{1-3}$. Recently, triazine and its derivatives have been regarded as efficient flame-retardant materials and good charring agents, mainly because that (i) they contain the stable triazine ring and can form the high-quality char layer during burning ${ }^{4,5}$; (ii) they are of beneficial merit as commercially available, comparatively low cost, and environmentally clean materials. However, the flame retardants having triazine ring, which are applied to some polymers, have been still not studied sufficiently $^{6-9}$. Therefore, these strongly enable us to prepare the triazine-containing flame retardant with the sulfur-nitrogen synergistic effect (defined as sulfur-nitrogen flame retardant containing triazine ring $)^{10,11}$ and apply it to the flame-retardant treatment of cotton.

In this paper, a sulfur-nitrogen flame retardant containing triazine ring ( $\mathrm{SN}$ ) has been successfully synthesized by the reaction of cyanuric chloride with sodium 2-aminoethanesulfanilate followed by diethanolamine. Its molecular structure was structurally characterized by elemental analysis, IR and ${ }^{1} \mathrm{H}$ NMR spectra. Meanwhile, the thermal behaviors of $\mathrm{SN}$, pure cotton, and cotton treated with SN were studied by thermogravimetry analysis (TGA). This result showed that SN was a good charring agent, and the char-forming ability and the thermal stability of cotton treated with SN were obviously improved.

\section{EXPERIMENTAL}

Cyanuric chloride and sodium 2-aminoethanesulfanilate were purchased from the Yingkou Sanzheng Organic Chemical Industry Company. Diethanolamine, acetone, and sodium carbonate were obtained from the Tianda Chemical Reagent Factory. All the starting materials and solvents were commercially available and used without further purification. $100 \%$ Cotton with density of $184 \mathrm{~g} / \mathrm{m}^{2}$ was supplied by Santai Fire Resistant Products Co., Ltd. The intermediate $\mathrm{M}$ was prepared according to the reported literature ${ }^{12}$.

Synthesis of SN: The target compound SN was prepared as shown in Scheme-I. In a typical experiment, the intermediate $\mathrm{M}(10 \mathrm{mmol})$ was dissolved to a $60 \mathrm{~mL}$ water at room temperature in $250 \mathrm{~mL}$ four-neck flask, and then the mixture reaction was warmed to $40{ }^{\circ} \mathrm{C}$. After stirring for $0.5 \mathrm{~h}$ at this temperature, the aqueous solution of diethanolamine (12 mmol) was slowly dropped into the above reaction vessel for $0.5 \mathrm{~h}$, in which $20 \%$ aqueous solution of sodium carbonate was added in order to keep $\mathrm{pH}=8-9$. The reaction process was monitored by thin layer chromatography (TLC) on silica 


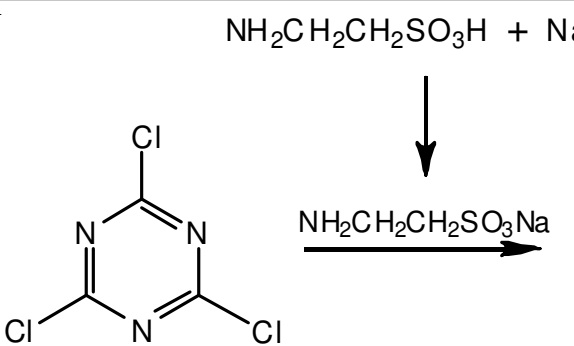

$\mathrm{C}$

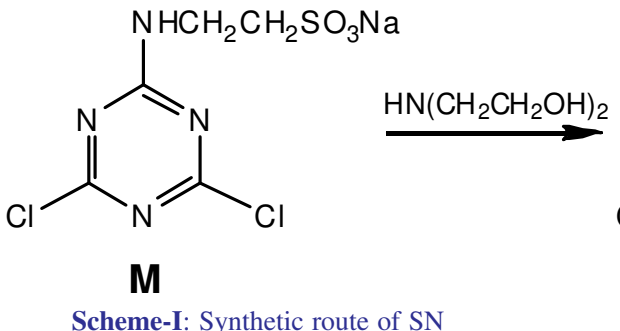<smiles>O=S(=O)(O)OCCNc1nc(Cl)nc(NCCO)n1</smiles>

SN gel eluenting with methanol-toluene $(2: 3, \mathrm{v}: \mathrm{v})$. When the reaction was completed, the reaction solvent was removed on a rotary evaporator. The above-obtained crude product was washed with anhydrous ethanol for three times and dried at room temperature. Finally, the target product was afforded as a white powder (Yield: $85 \%$ ). m.p. $>250{ }^{\circ} \mathrm{C}$; Anal. Calcd (\%) for $\mathrm{C}_{9} \mathrm{H}_{15} \mathrm{ClN}_{5} \mathrm{NaO}_{5} \mathrm{~S}$ : C 29.72, H 4.16, N 19.25; Found (\%): C $29.63, \mathrm{H} 4.28, \mathrm{~N} 19.11 .{ }^{1} \mathrm{H}$ NMR $\left(400 \mathrm{MHz}, d_{6}\right.$-DMSO, TMS, $\delta / \mathrm{ppm}): 8.90$ (s, 1H, NH), 5.26, $4.81(2 \mathrm{~s}, 2 \mathrm{H}, \mathrm{OH})$, 3.68-3.46 (m, 8H, $\left.\mathrm{NCH}_{2} \mathrm{CH}_{2} \mathrm{O}\right), 3.36$ (s, 2H, $\mathrm{NHCH}_{2}$ ), 2.98$3.00\left(\mathrm{~m}, 2 \mathrm{H}, \mathrm{CH}_{2} \mathrm{SO}_{3} \mathrm{Na}\right)$; IR $\left(\mathrm{KBr}, \mathrm{v}_{\max }, \mathrm{cm}^{-1}\right): 3524(\mathrm{NH})$, $3340(\mathrm{OH}), 1641$ (triazine ring), $1253(\mathrm{C}-\mathrm{N}), 1209,1074$ $(\mathrm{S}=\mathrm{O})^{13}$.

Flame-retardant treatment of cotton fabrics with $\mathrm{SN}$ : The general procedure is as follows: (i) cotton fabrics were washed in water with $0.5 \%$ (w/w) $\mathrm{NaOH}$ solution and then dried; (ii) aqueous flame-retardant finishing bath was prepared by various concentrations of $\mathrm{SN}$ at $90{ }^{\circ} \mathrm{C}$, where the liquor ratio was 1:20 and the $\mathrm{pH}$ was adjusted using sodium carbonate after adding sodium sulfate as a neutral salt; (iii) when the above-mentioned process was completed for about $1.5 \mathrm{~h}$, the cotton fabrics with $\mathrm{SN}$ were washed in the tap water and dried at $60{ }^{\circ} \mathrm{C}$ in an oven.

\section{RESULTS AND DISCUSSION}

\section{Optimal synthetic conditions}

Influence of reaction solvent on the yield of $\mathrm{SN}$ : The influence of solvent on the reaction to yield of SN was first considered for the optimal synthetic conditions. As the molar ratio of intermediate $\mathrm{M}$ and diethanolamine is 1 to 1 in the presence of sodium carbonate as acid-binding agent at $45^{\circ} \mathrm{C}$, we investigate the influence of several reaction solvents on the yield of SN and the results are presented in Table-1.

\begin{tabular}{|ccccc}
\multicolumn{5}{c}{ TABLE-1 } \\
\multicolumn{5}{|c}{ EFFECT OF REACTION SOLVENT ON THE YIELD OF SN } \\
\hline Solvent & Water & Water-Acetone & Acetone & Water-Ethanol \\
\hline Yield (\%) & 85 & 74.1 & 57.6 & 46.6 \\
\hline
\end{tabular}

As shown in Table-1, the maximum yield of $\mathrm{SN}$ reaches $85 \%$ when the solvent is water. This is possibly because that the starting materials (namely, intermediate $\mathrm{M}$ and diethanolamine) display better solubility in water than in common organic solvents such as water and ethanol, resulting in the homogeneous reaction system. Furthermore, it is noted that water is regarded as an environmentally-friendly solvent. Therefore, we selected water as the optimal reaction solvent.
Influence of acid-binding agent on the yield of $\mathrm{SN}$ : As the molar ratio of intermediate $\mathrm{M}$ and diethanolamine is 1 to 1 in water at $45^{\circ} \mathrm{C}$, we study the influence of acid-binding agent on the yield of SN and the results are listed in Table-2. Particularly, the inorganic bases are only selected as acid-binding agent, mainly because that (i) from the principle of the chemical equilibrium shift, the addition of the appropriate acid-binding agent can efficiently accelerate the reaction if hydrogen chloride is produced during the reaction; (ii) the common acidbinding agents contain the inorganic bases (e.g., sodium hydroxide and sodium carbonate, etc) and organic bases (e.g., pyridine and triethylamine, etc), where the inorganic bases are the best choice as they are lower-cost and environmentally-friendly.

Table- 2 showed the maximum yield of SN reached $85 \%$ when the acid-binding agent is sodium carbonate. This may be because that sodium hydroxide is more basic than sodium carbonate, which causes the appearance of much more side reaction. Thus, we chose sodium carbonate as the optimal acidbinding agent.

\begin{tabular}{ccc}
\multicolumn{3}{c}{ TABLE-2 } \\
\multicolumn{3}{c}{ EFFECT OF ACID-BINDING ON THE YIELD OF SN } \\
\hline Acid-binding agent & Sodium Carbonate & Sodium Hydroxide \\
Yield $(\%)$ & 85 & 68.6 \\
\hline
\end{tabular}

Influence of reaction temperature on the yield of $\mathrm{SN}$ : As the molar ratio of intermediate $\mathrm{M}$ and diethanolamine is 1 to 1 in the presence of sodium carbonate as acid-binding agent in water, the influence of reaction temperature on the yield of $\mathrm{SN}$ is investigated and the results are displayed in Fig. 1.

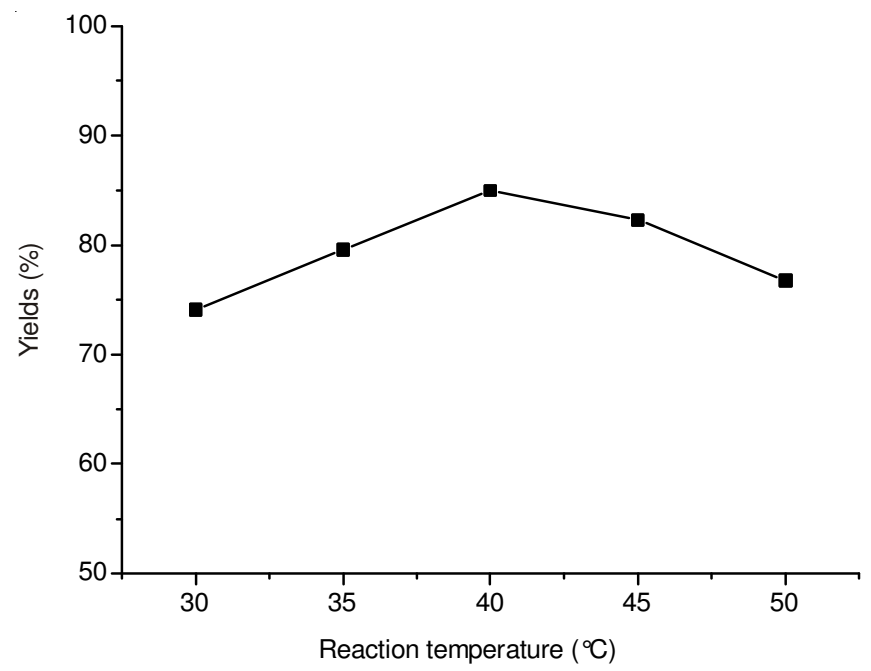

Fig. 1. Influence of reaction temperature on the yield of SN 
Fig. 1 shows that different reaction temperature hve the certain effect on the yield of $\mathrm{SN}$, in which the yield of $\mathrm{SN}$ reached the maximum when the reaction temperature was $40{ }^{\circ} \mathrm{C}$. This is mainly because that (i) for the reactivity of three chlorine atoms of cyanuric chloride, the first chlorine atom can react at $0-5{ }^{\circ} \mathrm{C}$ and the second chlorine atom can react at $40-60{ }^{\circ} \mathrm{C}^{14}$; (ii) for the most reaction, the increase of temperature can enhance the probability of the intermolecular effective collision, resulting in the rise of the reaction rate. Consequently, the optimal reaction temperature was at $45^{\circ} \mathrm{C}$.

Influence of $\mathbf{n}$ (intermediate I): $\boldsymbol{n}$ (diethanolamine) on the yield of SN: With the condition of water as reaction solvent, sodium carbonate as acid-binding agent, and reaction temperature at $40{ }^{\circ} \mathrm{C}$, the influence of the molar ratio of intermediate $\mathrm{M}$ to diethanolamine on the yield of $\mathrm{SN}$ is studied and the results are shown in Fig. 2.

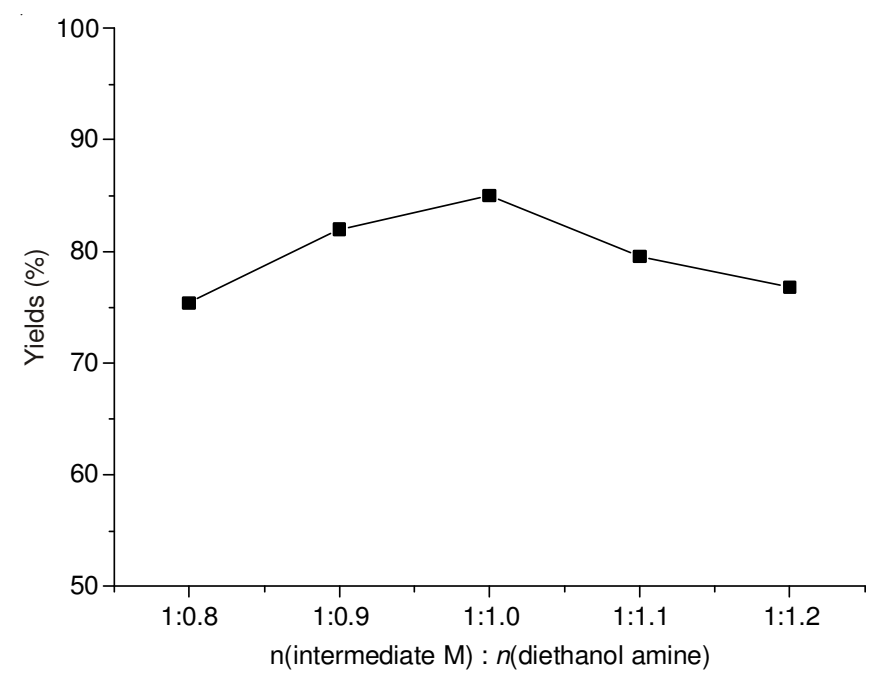

Fig. 2. Influence of $\mathrm{n}$ (intermediate $\mathrm{M}$ ): $n$ (diethanolamine) on the yield of SN

Fig. 2 displays that the yield of SN gradually increased when the range of the molar ratio of intermediate $M$ to diethanolamine is from 1:0.8 to 1:1.0 but sharply decreased as that of them is 1 to 1.2. Especially considering the cost of starting materials and the pollution of environment, we finally selected $n$ (intermediate $\mathrm{M}): n$ (diethanolamine $)=1: 1.0$ as the optimal molar ratio of starting materials.

Based on the above results, it is concluded for the optimal synthetic conditions that the yield of SN reaches $85 \%$ as the molar ratio of intermediate $\mathrm{M}$ to diethanolamine is 1 to 1 in the presence of sodium carbonate as acid-binding agent in water at $40{ }^{\circ} \mathrm{C}$.

Thermal decomposition behaviors: Fig. 3 displayed TGA (a) and DTG (b) curves of SN, pure cotton, cotton treated with 10 wt. \% SN under an air atmosphere, respectively. The TGA and DTG data were listed in Table-3, which included the initial decomposition temperature $\left(\mathrm{T}_{\text {onset }}\right)$, the temperature of maximum mass loss rate $\left(\mathrm{T}_{\max }\right)$ and the residue of the materials at $600{ }^{\circ} \mathrm{C}$. As shown in Table-3, for SN, its thermal degradation starting at $127.7^{\circ} \mathrm{C}$ was one-step decomposition and a large amount of charred residue $(46.8 \%)$ was left at $600{ }^{\circ} \mathrm{C}$, demonstrating that SN was an efficient char-forming agent. Comparatively, the thermal degradation of pure cotton, which undergone two-step degradation stages, started at 225.1, but it
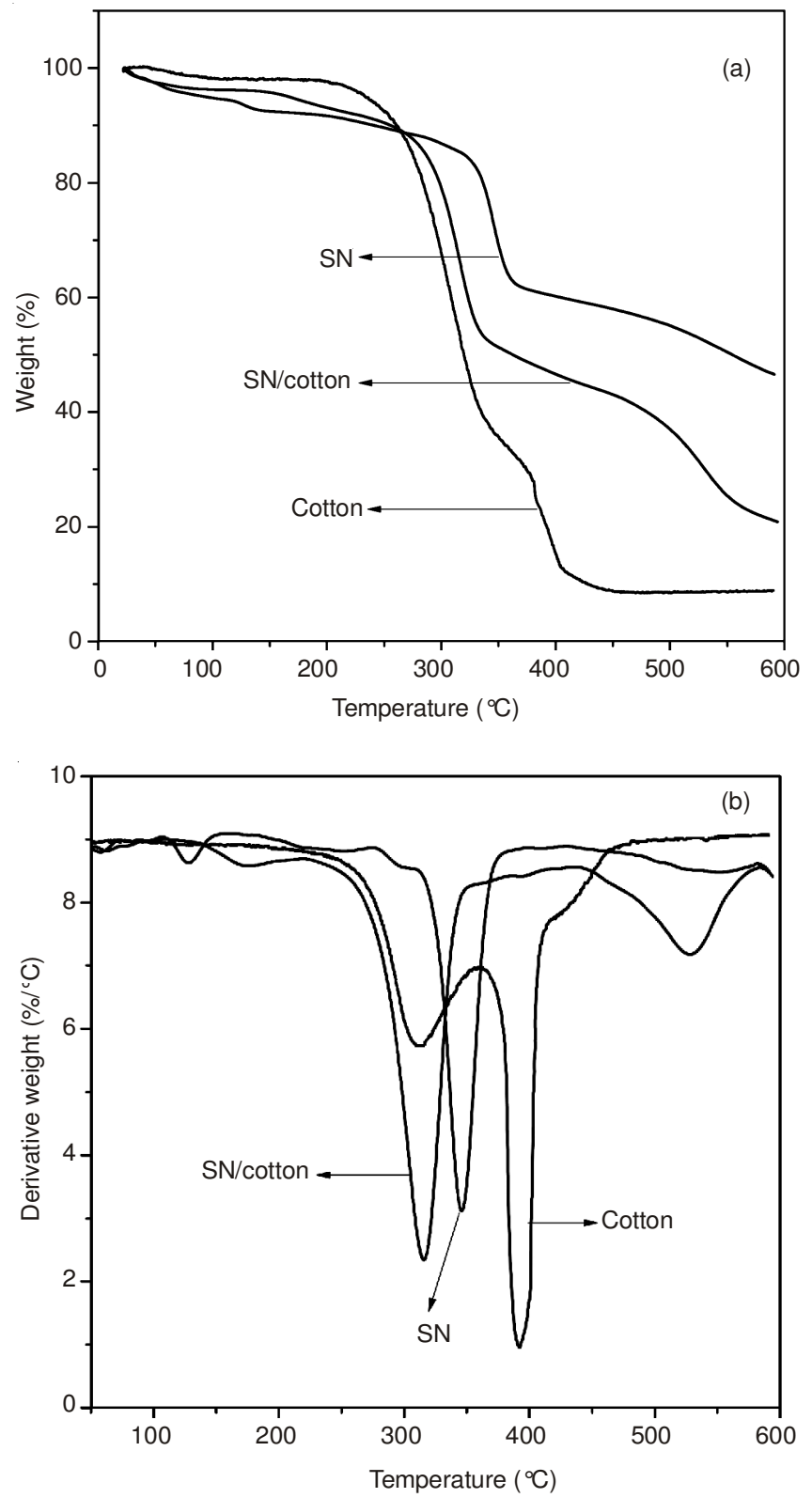

Fig. 3. TGA (a) and DTG (b) curves of SN, pure cotton, cotton treated with 10 wt. $\% \mathbf{S N}$ in air, respectively

\begin{tabular}{|c|c|c|c|c|}
\hline \multicolumn{5}{|c|}{$\begin{array}{l}\text { TABLE-3 } \\
\text { TGA AND DTG DATA OF SN, PURE COTTON, } \\
\text { COTTON TREATED WITH } 10 \text { wt. \% SN }\end{array}$} \\
\hline \multirow{2}{*}{ Samples } & \multirow{2}{*}{$\begin{array}{l}\mathrm{T}_{\text {onset }} \\
\left({ }^{\circ} \mathrm{C}\right)\end{array}$} & \multicolumn{2}{|c|}{$\mathrm{T}_{\max }\left({ }^{\circ} \mathrm{C}\right)$} & \multirow{2}{*}{$\begin{array}{c}\text { Residue } \\
\text { at } 600{ }^{\circ} \mathrm{C}(\%)\end{array}$} \\
\hline & & Stage 1 & Stage 2 & \\
\hline SN & 127.7 & 345.9 & - & 46.5 \\
\hline Cotton & 225.1 & 310.8 & 391.8 & 8.8 \\
\hline Cotton/SN & 166.6 & 315.6 & 528.7 & 21.4 \\
\hline
\end{tabular}

had only about $8.8 \%$ charred residue under the same condition. The initial decomposition temperature $\left(\mathrm{T}_{\text {onset }}=166.6^{\circ} \mathrm{C}\right)$ of cotton treated with $10 \mathrm{wt}$. \% SN was lower than that $\left(\mathrm{T}_{\text {onset }}=\right.$ $225.1^{\circ} \mathrm{C}$ ) of pure cotton, which might be attributed to the low Tonset of SN. Meanwhile, it could be found that the charred residue of cotton treated with $10 \mathrm{wt}$. $\% \mathrm{SN}$ was about $21.4 \%$ which was higher than that of pure cotton, possibly because 
that the sulfur-nitrogen synergistic effect in flame-retardant system had significant contribution to the char-forming ability and thermal stability of cotton ${ }^{15}$. These results indicated that SN not only changed the thermal degradation behavior of cotton but also promoted the char layer formation of cotton surface.

\section{Conclusion}

A novel sulfur-nitrogen flame retardant containing triazine ring $(\mathrm{SN})$ has been successfully synthesized and structurally characterized by means of elemental analysis, IR and ${ }^{1} \mathrm{H}$ NMR spectra. Experiments for the optimal synthetic conditions showed that the yield of $\mathrm{SN}$ reaches $85 \%$ when the molar ratio of intermediate $\mathrm{M}$ to diethanolamine is 1 to 1 in the presence of sodium carbonate as acid-binding agent in water at $40{ }^{\circ} \mathrm{C}$. In addition, TGA tests of $\mathrm{SN}$, pure cotton, cotton treated with 10 wt. \% SN have indicated that (i) SN was an efficient charforming agent, possibly due to the presence of the thermostable-triazine ring and the tertiary-nitrogen atom ${ }^{16}$; (ii) charforming ability of cotton treated was much higher than that of cotton untreated, which may be ascribed to the sulfur-nitrogen synergistic effect in the flame-retardant system composed of cotton fabrics and SN.

\section{ACKNOWLEDGEMENTS}

This work was financially supported by the National Natural Science Foundation of China (No. 21301160) and the Key Project of Science and Technology of Shanxi Province (No. 20120321016-02).

\section{REFERENCES}

1. S. Zhang and R. Horrocks, Prog. Polym. Sci., 28, 1517 (2003).

2. A.R. Horrocks, B.K. Kandola, P.J. Davies, S. Zhang and S.A. Padbury, Polym. Degrad. Stab., 88, 3 (2005).

3. W.D. Wu and C.Q. Yang, Polym. Degrad. Stab., 85, 623 (2004).

4. S.C. Chang, B. Condon, E. Graves, M. Uchimiya, C. Fortier, M. Easson and P. Wakelyn, Fiber. Polym., 12, 334 (2011).

5. J.D. Zuo, S.M. Liu and Q. Sheng, Molecules, 15, 7593 (2010).

6. S.S. Mahapatra and N. Karak, Polym. Degrad. Stab., 92, 947 (2007).

7. X. Wang, Y. Hu, L. Song, W.Y. Xing, H.D. Lu, P. Lv and G.X. Jie, Polym. Adv. Technol., 22, 2480 (2011).

8. K.H. Lee, D.H. Shin and Y.H. Seo, Korean J. Chem. Eng., 23, 224 (2006).

9. X.J. Lai, X.R. Zeng, H.Q. Li, F. Liao, C. Yin and H. Zhang, Polym. Compos., 33, 35 (2012).

10. C.S. Zhao, F.L. Huang, W.C. Xiong and Y.-Z. Wang, Polym. Degrad. Stab., 93, 1188 (2008).

11. S.V. Levchik and E.D. Weil, Polym. Int., 54, 981 (2005).

12. S.F. Zhao, Z.Y. Hu, H.L. Zhu and X. Li, Chin. J. Shandong Chem., 39, 3 (2010).

13. C.M. Feng, Y. Zhang, S.W. Liu, Z. Chi and J. Xu, J. Appl. Polym. Sci., 123, 3208 (2012).

14. J.F. Dai and B. Li, J. Appl. Polym. Sci., 116, 2157 (2010).

15. P.H. Zhao, M. Zhang, D.H. Wu and Y.Q. Liu, Korean J. Chem. Eng., 30, 1687 (2013).

16. C.H. Ke, J. Li, K.Y. Fang, Q.-L. Zhu, J. Zhu, Q. Yan and Y.-Z. Wang, Polym. Degrad. Stab., 95, 763 (2010). 